\begin{tabular}{|c|l|}
\hline Title & Extension of Traveling-Wave Parametric A mplifier Theory \\
\hline Author(s) & Sakuraba, Ichiro \\
\hline Citation & Memoirs of the Faculty of Engineering, Hokkaido University, 11(4), 435-446 \\
\hline Issue Date & 1963-03 \\
\hline Doc URL & http://hdl.handle.net/2115/37840 \\
\hline Type & bulletin (article) \\
\hline File Information & 11(4)_435-446.pdf \\
\hline
\end{tabular}

Instructions for use 


\title{
Extension of Traveling-Wave Parametric Amplifier Theory*
}

\author{
Ichiro SAKURABA
}

Department of Electronic Engineering

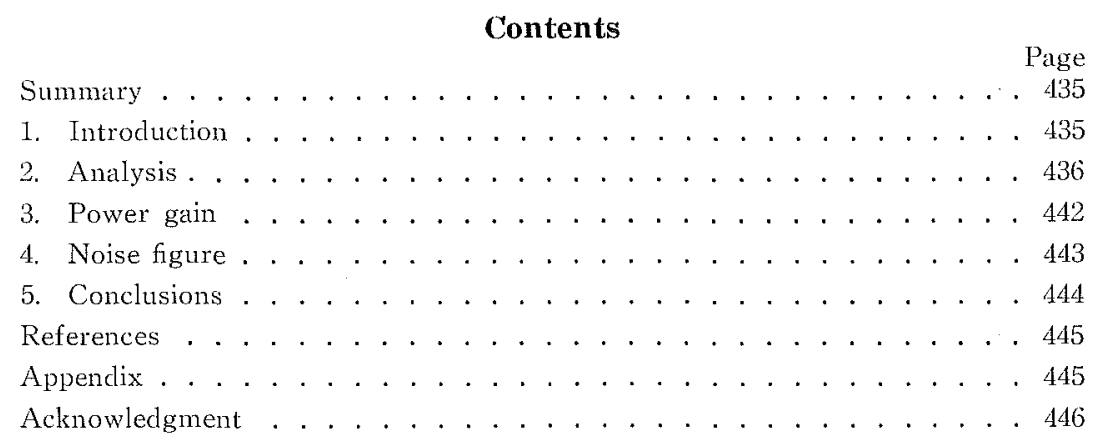

\section{Summary}

This paper presents an analysis of the traveling-wave parametric amplifier in which the signal, the first upper and lower sidebands around the fundamental pump frequency and the second lower sideband around the second pump harmonic are coupled together. The dervation of the equations was based on the usual traveling-wave parametric amplifier model of Tien and Suhl. The theoritical results are much closer to the measurements than were the results of the work presented by previous workers.

\section{Introduction}

A great deal of attention currently is being given to increasing the bandwidth capabilities of parametric amplifiers using ferromagnetic materials, junction diodes and space-charge waves by application of traveling-wave circuit techniques. In many respects, this situation is reminiscent of the evolution of broad band traveling-wave tubes from Klystron amplifiers employing resonant cavities. The analyses ${ }^{1-5)}$ of the traveling-wave parametric amplifier were previously investigated using a model in which coupling between only the first lower sideband and the signal was considered. However, the basic principles of parametric operation generate many sidebands at different frequencies. $T$. Makimoto ${ }^{6}$ explains a brief effect of including multi-frequency coupling on the

* Received September 29, 1962. 
amplification. J.S. Cook and W. H. Louisell' show the qualitative effect on the performance. S. Saito ${ }^{8)}$ investigated numerical calculations of the effect of the higher harmonic components on the amplification and noise figure of the longitudinal-beam type of parametric amplifier after some modifications of Haus' formula, but the result is restricted to the case where the signal approximately equals the first lower sideband. H. Sobol ${ }^{9)}$ presented an analysis of the longitudinal-beam parametric amplifier in which coupling is allowed between the signal, the lower and upper sidebands around the fundamental pump frequency, but his calculations are for the degenerate case in which the signal equals the lower sideband, and he did not analyse the noise figure. In the designing of slow wave circuits or loaded transmission lines, it is important that the effect of including higher idler components on the power gain and the noise fignre be given by a general formula. Then it may be shown that deterioration of the performance can easily be given after some modifications ${ }^{10-12)}$ of Tien's analysis.

\section{Analysis}

The analysis in which more idler frequencies are taken into account is given under the following assumptions:

1) The signal $\left(\omega_{1}\right)$ and all its higher harmonics $\left(\omega_{2}, \omega_{3} \cdots\right)$ are so small that the small signal theory can be applied.

2) The pumping wave $(\omega)$ with a propagation constant, $\beta$, has a power level substantially larger than that of the signal and its harmonics, and is so small that the second order coupling between the signal and the pumping waves can be neglected.

3) The signal $\left(\omega_{1}\right)$, the first lower sideband wave or idler $\left(\omega_{2}\right)$, the first upper sideband wave $\left(\omega_{3}\right)$, and the second lower sideband wave $\left(\omega_{4}\right)$ are carried by four propagating circuits. The relation of these frequencies is shown in Fig. 1.

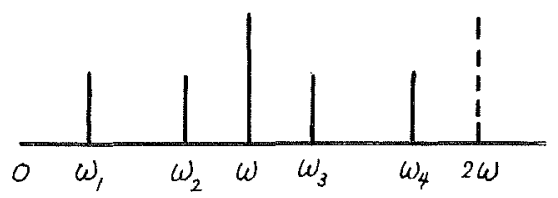

Fig. 1. Relation of pump, signal and idler frequencies. The frequencies satisfy $\omega=\omega_{1}+\omega_{2}, \quad \omega=\omega_{3}-\omega_{1}$ and $\omega_{4}=2 \omega-\omega_{1}$.

4) Four waves are coupled through a distributed inductance

$$
L_{(z, t)}=L_{(z, t)} e^{j \omega t}+L_{(z, t)}^{*} e^{-j \omega t}=L\left[e^{j(\omega t-\beta, z)}+e^{-j(\omega t-\beta z)}\right]
$$

Here $*$ denotes the complex conjugate, $t$ is time and $z$ is distance. 
A variable reacter such as the one shown in equation (1), may be obtained by feeding a pumping wave to a nonlinear medium in such a way that the equivalent permeability or dielectric of the medium varies with the intensity of the pumping field. The medium may be a ferrite sample, an electron beam or a series of junction diodes. The pumping wave may be supported by a separate propagation structure or by one of the circuits which carry the $\omega_{1}$, $\omega_{2}, \omega_{3}$ and $\omega_{4}$ waves.

5) The phase and group velocities of all waves face the same direction.

6) The conditions on the phases are

$$
\beta_{2}=\beta-\beta_{1}, \quad \beta_{3}=\beta+\beta_{1}, \quad \beta_{4}=2 \beta \cdots \beta_{1},
$$

where $\beta_{1}, \beta_{2}, \beta_{3}$ and $\beta_{4}$ are the phase constants of $\omega_{1}, \omega_{2}, \omega_{3}$ and $\omega_{4}$, respectively.

7) The transmission loss is small and may be neglected.

8) After dividing the propagating circuit into small sections, each section of the circuit is represented by a filter type network as shown in Fig. 2.

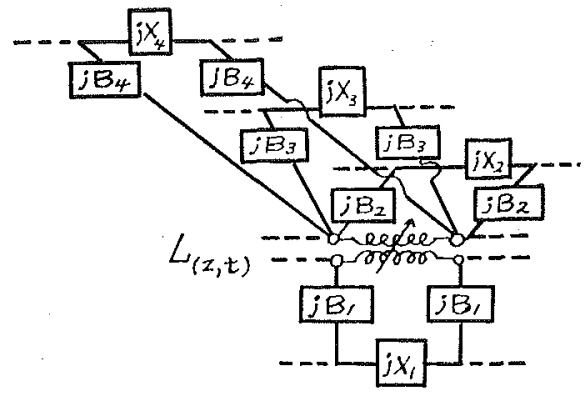

Fig. 2. An equivalent circuit of a traveling-wave parametric circuit with higher idler frequencies.

If the $i$ circuit has a series reactance $j X_{i}$ and a shunt susceptance $j B$, the phase constant and the charactristic impedance of the propagating circuit are,

$$
\beta_{i}=\left(X_{i} B_{i}\right)^{1 / 2}, \quad Z_{0 i}=\left(X_{i} / B_{i}\right)^{1 / 2}, \quad i=1,2,3,4 .
$$

If $V_{i(z, t)}$ and $I_{i(z, t)}$ are the voltage and current of the $i$ circuit, the differential equations for line are as follows;

$$
\left.\begin{array}{l}
\frac{\partial V_{1(z, t)}}{\partial z}=-j X_{1} I_{1(z, t)}-\frac{\partial}{\partial t}\left\{L_{(z, t)}\left[I_{2(z, t)}+I_{3(z, t)}+I_{4(z, t)}\right]\right\} \\
\frac{\partial I_{1(z, t)}}{\partial z}=-j B_{1} V_{1(z, t)} \\
\frac{\partial V_{2(z, t)}}{\partial z}=-j X_{2} I_{2(z, t)}-\frac{\partial}{\partial t}\left\{L_{(z, t)}\left[I_{1(x, t)}+I_{3(z, t)}+I_{4(z, t)}\right]\right\}
\end{array}\right\}
$$




$$
\begin{aligned}
& \frac{\partial I_{2(z, t)}}{\partial z}=-j B_{2} V_{2(z, t)} \\
& \frac{\partial V_{3(z, t)}}{\partial z}=-j X_{3} I_{3(z, t)}-\frac{\partial}{\partial t}\left\{L_{(z, t)}\left[I_{1(z, t)}+I_{2(z, t)}+I_{4(z, t)}\right]\right\} \\
& \frac{\partial I_{3(z, t)}}{\partial z}=-j B_{3} V_{3(z, t)} \\
& \frac{\partial V_{4(z, t)}}{\partial z}=-j X_{4} I_{4(z, t)}-\frac{\partial}{\partial t}\left\{L_{(z, t)}\left[I_{1(z, t)}+I_{2(z, t)}+I_{3(z, t)}\right]\right\} \\
& \frac{\partial I_{4(z, t)}}{\partial z}=-j B_{4} V_{4(z, t)}
\end{aligned}
$$

Combination of the first equation with the second, the third with the fourth and the fifth with the sixth yields

$$
\left.\begin{array}{l}
\frac{\partial^{2} I_{1(z, t)}}{\partial z^{2}}=-X_{1} B_{1} I_{1(z, t)}+j B_{1} \frac{\partial}{\partial t}\left\{L_{(z, t)}\left[I_{2(z, t)}+I_{3(z, t)}+I_{4(z, t)}\right]\right\} \\
\frac{\partial^{2} I_{2(z, t)}}{\partial z^{2}}=-X_{2} B_{2} I_{2(z, t)}+j B_{2} \frac{\partial}{\partial t}\left\{L_{(z, t)}\left[I_{1(z, t)}+I_{3(z, t)}+I_{4(z, t)}\right]\right\} \\
\frac{\partial^{2} I_{3(z, t)}}{\partial z^{2}}=-X_{3} B_{3} I_{3(z, t)}+j B_{3} \frac{\partial}{\partial t}\left\{L_{(z, t)}\left[I_{1(z, t)}+I_{2(z, t)}+I_{4(z, t)}\right]\right\} \\
\frac{\partial^{2} I_{1(z, t)}}{\partial z^{2}}=-X_{4} B_{4} I_{4(z, t)}+j B_{4} \frac{\partial}{\partial t}\left\{L_{(z, t)}\left[I_{1(z, t)}+I_{2(z, t)}+I_{3(z, t)}\right]\right\}
\end{array}\right\}
$$

These are familiar equations except for the coupling terms. We shall assume the coupling terms to be small, compared with the other terms in the equations so that calculation can be made by the perturbation method. We should point out that equation (4) is derived by assuming $I_{i(z, t)}$ to be in the form $A_{i(z)} \exp j \omega_{i} t$, if $I_{i(z, t)}=A_{i(z)}^{*} \exp (-j \omega t)$ the sign of the coupling term in equation (4) is reversed. In the following assumptions, let the conditions on the phase constants and frequencies be

$$
\begin{aligned}
& \omega_{2}=\omega-\omega_{1}, \quad \omega_{3}=\omega+\omega_{1}, \quad \omega_{4}=2 \omega-\omega_{1}, \\
& \beta_{2}=\beta-\beta_{1}, \quad \beta_{3}=\beta+\beta_{1}, \quad \beta_{4}=2 \beta-\beta_{1} .
\end{aligned}
$$

The complete solution of equation (4) must be the sum of coupled pairs. Here for simplification, we shall analyse the next pair only. Put

$$
\begin{aligned}
& I_{1(z, t)}=A_{1(z)} e^{j\left(\omega_{1} t-\beta_{1} z\right)} \\
& I_{2(z, t)}^{*}=A_{2(z)}^{*} e^{-j\left(\omega_{2} t-\beta_{2} z\right)} \\
& I_{3(z, t)}=A_{3(z)} e^{j\left(\omega_{3} t-\beta_{3} z\right)} \\
& I_{4(z, t)}^{*}=A_{4(z)}^{*} e^{-j\left(\omega_{4} t-\beta_{4} z\right)}
\end{aligned}
$$


From the following lines of approach similar to those described in the text of Tien and Suhl ${ }^{1}$, we obtain a set of equations

$$
\begin{aligned}
& \frac{\partial A_{1(z)}}{\partial z}=-j \frac{1}{2} \frac{\omega_{1} B_{1} L}{\beta_{1}}\left[A_{2(z)}^{*}+A_{3(z)}\right] \\
& \frac{\partial A_{2(z)}^{*}}{\partial z}=j \frac{1}{2} \frac{\omega_{2} B_{2} L}{\beta_{2}}\left[A_{1(z)}+A_{4(z)}^{*}\right] \\
& \frac{\partial A_{3(z)}}{\partial z}=-j \frac{1}{2} \frac{\omega_{3} B_{3} L}{\beta_{3}} A_{1(z)} \\
& \frac{\partial A_{4(z)}^{*}}{\partial z}=j \frac{1}{2} \frac{\omega_{4} B_{4} L}{\beta_{4}} A_{2(z)}^{*}
\end{aligned}
$$

It can easily be shown that equation (7) satisfies the Manley-Rowe power relationship, as shown in Appendix. Equation (7) may be reduced in the form

$$
\begin{aligned}
\frac{\partial^{4} A_{1(z)}}{\partial z^{4}} & +\frac{L^{2}}{4}\left(\frac{\omega_{1} \omega_{3}}{Z_{02} Z_{03}}+\frac{\omega_{2} \omega_{4}}{Z_{02} Z_{04}}-\frac{\omega_{1} \omega_{2}}{Z_{01} Z_{02}}\right) \frac{\partial^{2} A_{1(z)}}{\partial z^{2}} \\
& +\frac{L^{4}}{16} \frac{\omega_{1} \omega_{2} \omega_{3} \omega_{4}}{Z_{01} Z_{02} Z_{03} Z_{04}} A_{1(z)}=0
\end{aligned}
$$

For understanding the effect of higher harmonics, it is assumed that

$$
Z_{01} Z_{03}=Z_{02} Z_{04}=Z_{01} Z_{02}
$$

where $Z_{01}, Z_{02}, Z_{03}$ and $Z_{04}$ are the characteristic impedances of $\omega_{1}, \omega_{2}, \omega_{3}$ and $\omega_{4}$, respectively. Thus equation (8) can be written as

$$
\begin{aligned}
\frac{\partial^{4} A_{1(z)}}{\partial z^{4}} & +\frac{1}{4} \xi_{0}^{2}\left(3 n^{2}-3 n+2\right) \frac{\partial^{2} A_{1(z)}}{\partial z^{2}} \\
& +\frac{1}{16} \xi_{0}^{2}\left(1-n^{2}\right)(2-n) n A_{1(z)}=0
\end{aligned}
$$

where $\omega^{2} L^{2} / Z_{01} Z_{02}=\xi_{0}^{2}, \omega_{1} / \omega=n, 0.5 \geqq n>0$. Then the solution of equation (10) is

$$
A_{1(z)}=a_{1} e^{\hat{\delta}_{1} \hat{\xi}_{0} z}+b_{1} e^{\hat{j}_{2} \hat{\xi}_{0} z}+c_{1} e^{\hat{j}_{3} \hat{\hat{t}}_{0} z}+d_{1} e^{\hat{\delta}_{4} \hat{\epsilon}_{0} z}
$$

$\delta$ can be determined from equation (12),

$$
\delta^{4}+\frac{1}{4}\left(3 n^{2}-3 n+2\right) \delta^{2}+\frac{1}{16}\left(1-n^{2}\right)(2-n) n=0
$$

In equation (11), $a_{1}, b_{1}, c_{1}$ and $d_{1}$ are arbitrary constants. In dealing with equation (12), let

$$
\delta=x+j y
$$

From equation (11), we see that, if $x>0$, the wave increases as it travels, 


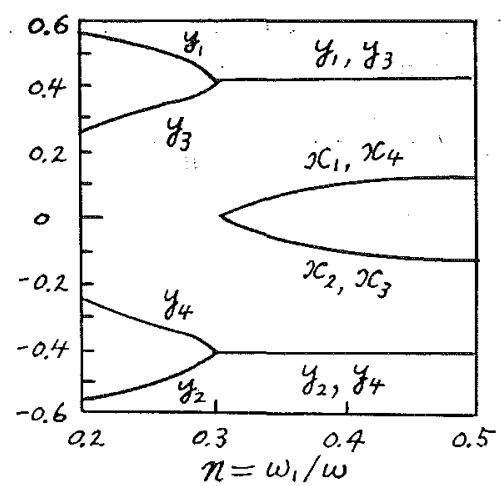

Fig. 3. The $x^{\prime}$ s and $y^{\prime}$ s for waves versus the ratio of the signal frequency to the fundamental pump frequency. Here the four waves vary with distance as $\exp \left(-j \beta+\delta_{0}^{\circ}\right) z$ where $\delta=x+j y$.

and if $x<0$, the wave decreases as it travels, and $y$ means the difference of phase constant after coupling. Curves of $\delta$ are shown in Fig. 3. Increasing waves are found for $0.5 \geqq n>0.303$. Maximum $x$ occurs under the degenerated case in which $n=0.5$. This coinsides with the results by Saito ${ }^{87}$ and Sobol ${ }^{9}$. For $n<0.303$, it is noted that all the coupled waves are neither growing nor attenuating.

Finally from equations (7) and (11) the following equations may be led

$$
\begin{aligned}
& I_{\mathrm{I}(z, t)}=e^{j\left(\omega_{1} t-\beta_{1} z\right)}\left(a_{1} e^{\delta_{1} \xi_{0} z}+b_{1} e^{-\delta_{1} \xi_{0} z}+c_{1} e^{\tilde{o}_{3} \xi_{0} z}+d_{1} e^{-\delta_{3} \xi_{0} z}\right) \\
& I_{2(z, t)}^{*}=e^{-j\left(\omega_{2} t-\beta_{2} z\right)}\left[M_{2}\left(a_{1} e^{\delta_{1} \xi_{0} z}-b_{1} e^{-\dot{\delta}_{1} \hat{\xi}_{0} z}\right)+N_{2}\left(c_{1} e^{\delta_{3} \xi_{0} z}-d_{1} e^{-\bar{\delta}_{3} \hat{s}_{0} z}\right)\right] \\
& I_{3(z, t)}=e^{j\left(\omega_{3} t-\hat{\beta}_{3} z\right)}\left[M_{3}\left(a_{1} e^{\delta_{1} \hat{\xi}_{0} z}-b_{1} e^{-\hat{j}_{1} \hat{\delta}_{0} z}\right)+N_{3}\left(c_{1} e^{\delta_{3} \hat{\xi}_{0} z}-d_{1} e^{-\hat{\delta}_{3} \hat{\xi}_{0} z}\right)\right] \\
& I_{4(z, t)}^{*}=e^{-j\left(\omega_{4} t-\hat{\beta}_{4} z\right)}\left[M_{4}\left(a_{1} e^{\hat{j}_{1} \hat{\xi}_{0} z}+b_{1} e^{-\delta_{1} \hat{\xi}_{0} z}\right)+N_{4}\left(c_{1} e^{\hat{\delta}_{3} \hat{\xi}_{0} z}+d_{1} e^{-\hat{o}_{3} \hat{\xi}_{0} z}\right)\right]
\end{aligned}
$$

where

$$
\begin{array}{ccc}
M_{2}=\left(\frac{\gamma_{3}}{\delta_{1} \xi_{0}}-\frac{\delta_{1} \xi_{0}}{\gamma_{1}}\right) & M_{3}=-\gamma_{3} / \delta_{1} \xi_{0} . \quad & M_{4}=\left(\frac{\gamma_{3}}{\delta_{1}^{2} \xi_{0}^{2}}-\frac{1}{\gamma_{1}}\right) \gamma_{4} \\
N_{2}=\left(\frac{\gamma_{3}}{\delta_{3} \xi_{0}}-\frac{\delta_{3} \xi_{0}}{\gamma_{1}}\right) & N_{3}=-\gamma_{3} / \delta_{3} \xi_{0} . \quad N_{4}=\left(\frac{\gamma_{3}}{\delta_{3}^{2} \xi_{0}^{2}}-\frac{1}{\gamma_{2}}\right) \gamma_{4} \\
\gamma_{i}=\frac{j}{2} \frac{\omega_{i} B_{i} L}{\beta_{i}}
\end{array}
$$

In the following case

$$
I_{1(0, t)}=a e^{j\left(\omega_{1} t+0\right)}, \quad I_{2(0, t)}^{*}=I_{3(0, t)}=I_{4(0, t)}^{*}=0 .
$$

For $0.5 \geqq n>0.303$, equation (14) becomes 


$$
\left.\begin{array}{rl}
I_{1(z, t)} & =e^{j\left(\omega_{1} t-\beta_{1} z+\theta\right)} \frac{a}{M_{4}^{*}-M_{4}}\left[M_{4}^{*} \cosh \left(\delta_{1} \xi_{0} z\right)-M_{4} \cosh \left(\delta_{1}^{*} \xi_{0} z\right)\right] \\
I_{2(z, t)}^{*} & =e^{-j\left(\omega_{2} t-\beta_{z} z-\theta\right)} \frac{a}{M_{4}^{*}-M_{4}}\left[M_{2} M_{4}^{*} \sinh \left(\delta_{1} \xi_{0} z\right)+M_{2}^{*} M_{4} \sinh \left(\delta_{1}^{*} \xi_{0} z\right)\right] \\
I_{3(z, t)} & =e^{j\left(\omega_{3} t-\beta_{3} z+\theta\right)} \frac{a}{M_{4}^{*}-M_{4}}\left[M_{2} M_{4}^{*} \sinh \left(\delta_{1} \xi_{0} z\right)+M_{3}^{*} M_{4} \sinh \left(\delta_{1}^{*} \xi_{0} z\right)\right] \\
I_{4(z, t)}^{*} & =e^{--j\left(\omega_{4} t-\beta_{4} z-\theta\right)} a \frac{M_{4} M_{4}^{*}}{M_{4}^{*}-M_{4}}\left[\cosh \left(\delta_{1} \xi_{0} z\right)-\cosh \left(\delta_{1}^{*} \xi_{0} z\right)\right]
\end{array}\right\}
$$

where

$$
N_{2}=M_{2}^{*}, \quad N_{3}=M_{3}^{*}, \quad N_{4}=M_{4}^{*} .
$$

The amplitudes of $I_{1(z, t)}, I_{2(z, t)}^{*}, I_{3(z, t)}$ and $I_{4(z, t)}^{*}$ are plotted in Fig. 4, which should be compared with coupling only between the signal and its lower
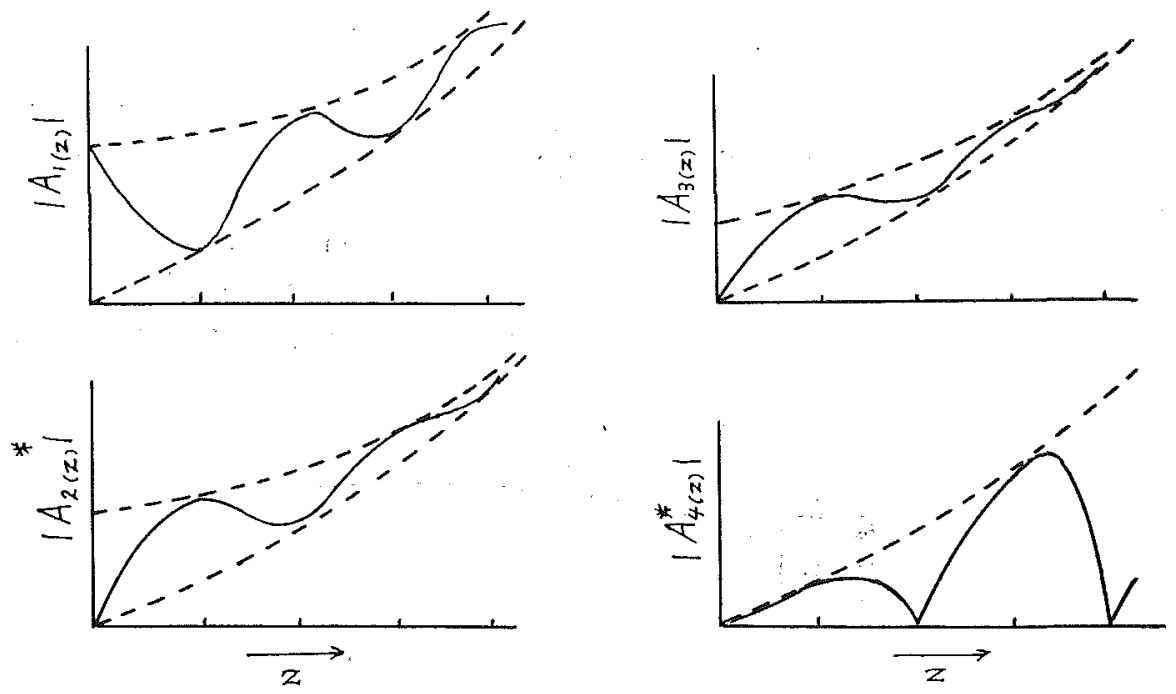

Fig. 4. The amplitudes of the $\omega_{1}, \omega_{2}, \omega_{3}$ and $\omega_{4}$ waves plotted along the direction of propagation.

sideband. It is noted that these forms resemble amplitude-modulated increasing waves and the amplitude of $I_{4(z, t)}^{*}$ is zero at $y_{1} \xi_{0} z=0, \pi, 2 \pi, \cdots$.

For $n<0.303$, we have

$$
\left.\begin{array}{l}
I_{1(z, t)}=e^{j\left(\omega_{1} t-\beta_{1} z+\theta\right)} \frac{a}{N_{4}-M_{4}}\left[N_{4} \cos \left(y_{1} \xi_{0} z\right)-M_{4} \cos \left(y_{1} \xi_{0} z\right)\right] \\
I_{2(z, t)}^{*}=e^{-j\left(\omega_{2} t-\beta_{2} z-\theta\right)} \frac{j a}{N_{4}-M_{4}}\left[M_{3} N_{4} \sin \left(y_{1} \xi_{0} z\right)-M_{4} N_{2} \sin \left(y_{1} \xi_{0} z\right)\right]
\end{array}\right\}
$$




$$
\begin{aligned}
& I_{3(z, t)}=e^{j\left(\omega_{3} t-\beta_{3} z+\theta\right)} \frac{j a}{N_{4}-M_{4}}\left[M_{3} N_{4} \sin \left(y_{1} \xi_{0} z\right)-M_{4} N_{3} \sin \left(y_{3} \xi_{0} z\right)\right] \\
& I_{4(z, t)}^{*}=e^{-j\left(\omega_{4} t-\beta_{4} z-\theta\right)} \frac{M_{4} N_{4}}{N_{4}-M_{4}}\left[\cos \left(y_{1} \xi_{0} z\right)-\cos \left(y_{3} \xi_{0} z\right)\right]
\end{aligned}
$$

\section{Power Gain}

It is assumed that a signal is applied at frequency $\omega_{1}$. From equation (17) it follows that the average power transmitted down the line exponentially increases and that gains can be achieved. The power gain in decibels in a length $s$ is given by

$$
\text { gain }(\mathrm{db})=20 \log \left(\cosh x_{1} \xi_{\mathrm{o}} s\right) .
$$

If the amplifier is long enough so that $x_{1} \xi_{0} s \gg 1$, the gain can be expressed approximately as

$$
\text { gain }(\mathrm{db}) \fallingdotseq 8.68 x_{1} \xi_{0} s-6 \mathrm{db}
$$

so that the maximum gain occurs when $n=0.5$. Here the second term, -6 , is a loss relating power associated with the growing wave to the total applied signal. The power gain in various conditions described above is plotted in Fig. 5. The curve (a) of Fig. 5 shows the gain when the signal, the idler, the first upper sideband and the second lower sideband are coupled together. For coupling only between the signal and the idler, the power gain is given

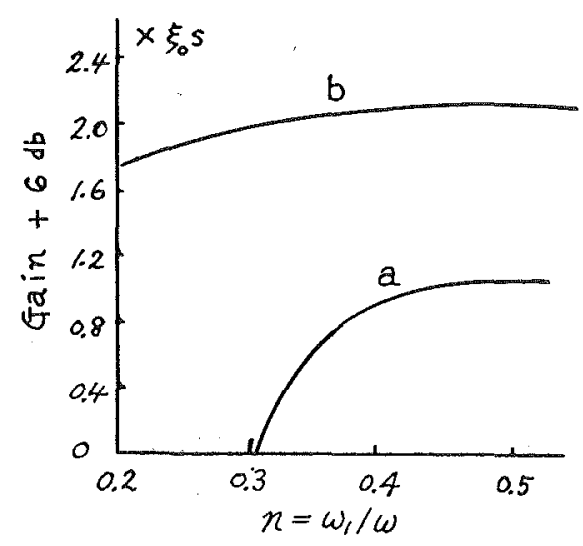

Fig. 5. Power gain of a traveling-wave parametric amplifier versus the ratio $n=\omega_{1} / \omega$, a) $\omega_{1}, \omega_{2}, \omega_{3}$ and $\omega_{4}$ waves are coupled together; b) coupling only between $\omega_{1}$ and $\omega_{2}$. At $n=0.5$, the effect of including the multifrequency coupling is to reduce the gain to one half the $\mathrm{db}$ value predicted by earlier works. 
by the curves (b) of Fig. 5, as shown by Tien ${ }^{3)}$. From the calculations described above, the effect of including the multi-frequency coupling is to reduce the gain to one half the $\mathrm{db}$ value predicted in earlier analyses. These theoritical analyses are much closer to the measurements than were the results of the earlier workers ${ }^{1-5)}$.

\section{Noise Figure}

The primary advantage of parametric amplifiers is their extremely low noise capabilities. A discussion of the noise figure of including the multifrequency coupling will now be presented.

It is assumed that the input is connected to a matched signal source at $T_{1}=290^{\circ} \mathrm{K}$ at frequency $\omega_{1}$. Further assume that at $\omega_{2}, \omega_{3}$ and $\omega_{4}$, the input ends are matched passive impedances at temperature $T_{2}, T_{3}$ and $T_{4}$, respectively, and the output ends are matched to a load at temperature $T_{s}$. This is shown

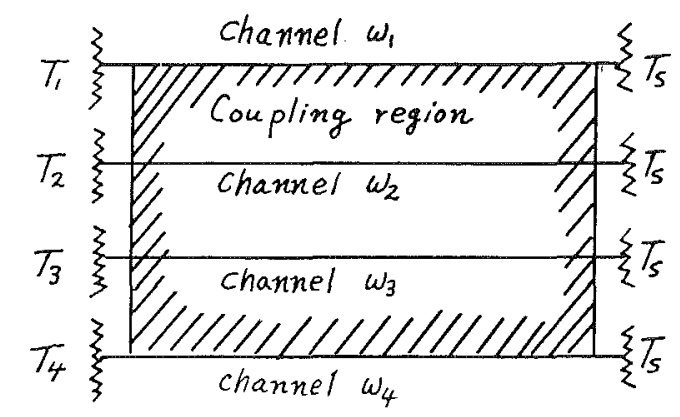

Fig. 6. Schematic representation of four channels for noise and signal in a traveling-wave parametric amplifier. The input of $\omega_{1}, \omega_{2}, \omega_{3}$ and $\omega_{4}$ channels are in matched impedances at respective temperature $T_{1}$, $T_{2}, T_{3}$ and $T_{4}$, and the outputs of their channels are terminated in matched impedances at temperature $T_{s}$.

schematically in Fig. 6. The pumping wave, signal, idler, the first upper and the second lower sidebands satisfy equations (5) and (6), so that the noise inputs, $k T_{1} B, k T_{2} B, k T_{3} B$ and $k T_{4} B$ of $\omega_{1}, \omega_{2}, \omega_{3}$ and $\omega_{4}$ circuits, respectively, give a noise output

$$
k T_{1} B+\frac{\omega_{1}}{\omega_{2}} k T_{2} B(G-1)+\frac{\omega_{1}}{\omega_{3}} k T_{3} B(G-1)+\frac{\omega_{1}}{\omega_{4}} k T_{4} B(G-1)
$$

at $z=s$ of the circuit, where $y_{1} \xi_{0} s \neq n \pi,(n=1,2,3, \cdots)$. If $y_{1} \xi_{0} s=n \pi$, the fourth term becomes zero.

If a signal, $S_{\mathrm{nn}\left(\omega_{1}\right)}$, is introduced at $\omega_{1}$ only, then from the definition of 
gain, $S_{\text {out }\left(\omega_{1}\right)}=G \cdot S_{\mathrm{in}\left(\omega_{1}\right)}$ and equation $(21)$, the noise figure, $F$, is

$$
F=1+\frac{\omega_{1}}{\omega_{2}} \frac{T_{2}}{T_{1}} \frac{G-1}{G}+\frac{\omega_{1}}{\omega_{3}} \frac{T_{3}}{T_{1}} \frac{G-1}{G}+\frac{\omega_{1}}{\omega_{4}} \frac{T_{4}}{T_{1}} \frac{G-1}{G}
$$

If $G \gg 1$, equation (22) becomes

$$
F \doteqdot 1+\frac{\omega_{1} T_{2}}{\omega_{2} T_{1}}+\frac{\omega_{1} T_{3}}{\omega_{3} T_{1}}+\frac{\omega_{1} T_{4}}{\omega_{4} T_{1}}
$$

When $T_{1}=T_{2}=T_{3}=T_{4}$, the noise figure is

$$
F=1+\frac{n}{1-n}+\frac{n}{1+n}+\frac{n}{2-n}
$$

Now, $\omega_{1}=\omega_{2}$, it is seen that the noise figure is $4.3 \mathrm{db}$, which is larger than that of the coupling only between the signal and idler by $1.3 \mathrm{db}$. This relation is shown in Fig. 7. The curve (a) of Fig. 7 gives the noise figure

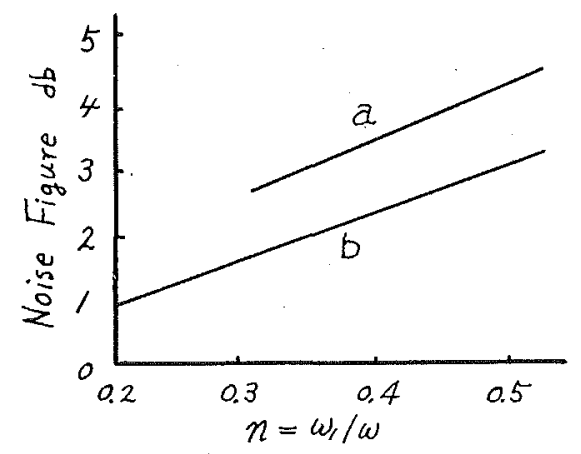

Fig. 7. Noise figure versus the ratio $n=\omega_{1} / \omega$, a) $\omega_{1}, \omega_{1}, \omega_{3}$ and $\omega_{4}$ waves are coupled together; b) coupling only between $\omega_{1}$ and $\omega_{2}$. At $n=0.5$, the effect of higher idler frequencies is to increase the noise figure to $4.3 \mathrm{db}$.

when four waves are coupled together. In the case of coupling only between. $\omega_{1}$ and $\omega_{2}$ the noise figure is shown by the curve (b) of Fig. 7, as given in Tien $^{3)}$. From calculations described above, the effect of including the multifrequency is to increase the noise figure. This result agrees with the noise figure of the longitudinal-beam type computed by Saito ${ }^{83}$, also.

\section{Conclusions}

An analysis in which the signal, the first upper sideband, the first lower sideband and the second lower sideband were coupled together was presented. 
The calculations in this paper yielded the following interesting results:

1) The deterioration of the performance, e.g. the amplification and the noise figure, was given in a general form. The dervation of the analysis was based on the usual model of traveling-wave parametric amplifier given by Tien and Suhl.

2) The effect of including the multi-frequency was to reduce the gain to one half the $\mathrm{db}$ value predicted by earlier analyses and to increase the noise figure to $4.3 \mathrm{db}$.

3) The theoritical results were much closer to the measurements than were the results of the earlier workers.

\section{References}

1) P. K. Tien and H. Suhl: "A traveling-wave feromagnetic amplifier", Proc. IRE, vol. 46, pp. 700-706; April, 1958.

2) W. H. Louisell and C.F. Quate: "Parametric amprification of space charge waves", Proc. IRE, vol. 46, pp. 707-716; April, 1958.

3) P.K. Tien: "Parametric amplification and frequency mixing in propagating circuits", J. Appl. Phys, vol. 29, pp. 1347-1357; September, 1958.

4) S. Saito: "Parametric amplification of space-charge waves on a thin electron beam, "J. Inst. Elect. Commun. Engrs. Japan, vol. 43, pp. 1113-1120; November, 1958 (in Japanes).

5) B. T. Henoch: "Aspects on wide-band parametric traveling-wave amplifiers", Ericsson Technics, vol. 16, pp. 77-135; January, 1960.

6) T. Makimoto: "Parametric amplification in propagating circuits", Microwave Transmission Research Committee, Inst. Elect. Commun. Engrs. Japan; May, 1959 (in Japanese).

7) J. S. Cook and W. H. Louisell: "Fast longitudinal space-charge wave parametric amplifier", 1959 WESCON Convention Record, pt. 3, pp. 77-85.

8) S. Saito: "Effect of higher harmonic components on the performance of the travelingwave parametric amplifier, "Proc. IRE, vol. 49, pp. 623-624; March, 1960.

9) H. Sobol: "Extension of longitudinal beam parametric amplifier theory", Proc. IRE, vol. 48, pp. 792-793; April, 1960.

10) Y. Asami and I. Sakuraba: "Gain and noise figure of traveling-wave parametric amplifiers", The Record of General Meeting, B9, Hokkaido Section of I. E. E. and I.E.C.E. of Japan; November, 1960 (in Japanese).

11) I. Sakuraba and K. Kazui: "Effect of higher idler frequencies on gain and noise figure of traveling-wave parametric amplifier, "J. Inst. Elect. Commun. Engrs. Japan, vol. 44, pp. 1803-1806; November, 1961 (in Japanese).

12) I. Sakuraba: "Extension of traveling-wave parametric amplification theory", Proc. IEEE, Vol. 51, pp. 371-372; February 1963. 


\section{Appendix}

The Manley-Rowe relation under small signal and weak coupling conditions are

$$
\frac{d}{d z}\left(\sum_{m=-\infty}^{\infty} \frac{a_{m} a_{m}^{*}}{m \omega+\omega_{1}}\right) d z=0
$$

or

$$
\sum_{m=-\infty}^{\infty} \frac{1}{m \omega+\omega_{1}}\left(a_{m} \frac{d a_{m}^{*}}{d z}+a_{m}^{*} \frac{d a_{m}}{d z}\right) d z=0
$$

where $a_{m}$ is the normal amplitude and $d z$ is length. The relation between the mode amplitude, $a_{m}$ and $A_{i(z)}$ of equation (7) is

$$
a_{m}=A_{i(z)} \sqrt{Z_{0 i}}
$$

Here $Z_{0 i}$ is the characteristic impedance of $\omega_{i}$ wave. The signal $(i=1)$, the idler $(i=2)$, the first upper sideband $(i=3)$, and the second lower sideband $(i=4)$ correspond to $m=0, m=-1, m=+1$ and $m=-2$, respectively. Then equation (7) becomes

$$
\begin{aligned}
& \frac{\partial a_{0}}{\partial z}=-j \frac{1}{2} \frac{\omega_{1} B_{1} L}{\beta_{1}} \sqrt{\frac{Z_{01}}{Z_{02} Z_{03}}}\left(a_{-1}^{*} \sqrt{Z_{03}}+a_{1} \sqrt{Z_{02}}\right) \\
& \frac{\partial a_{-1}^{*}}{\partial z}=j \frac{1}{2} \frac{\omega_{2} B_{2} L}{\beta_{2}} \sqrt{\frac{Z_{01}}{Z_{01} Z_{04}}}\left(a_{0} \sqrt{Z_{04}}+a_{-2}^{*} \sqrt{Z_{01}}\right) \\
& \frac{\partial a_{1}}{\partial z}=-j \frac{1}{2} \frac{\omega_{3} B_{3} L}{\beta_{3}} \sqrt{\frac{Z_{03}}{Z_{01}}} a_{0} \\
& \frac{\partial a_{-2}^{*}}{\partial z}=j \frac{1}{2} \frac{\omega_{4} B_{4} L}{\beta_{4}} \sqrt{\frac{Z_{04}}{Z_{03}}} a_{1}^{*}
\end{aligned}
$$

The equation (A-3) and its conjugate satisfy equation $(A-1)$. Thus it was shown that equation (7) satisfies the Manley-Rowe power relationship.

\section{Acknowledgment}

The author wishes to express his sincere appreciation to Dr. Y. Asami for several illuminating discussions on this problem, and to $\mathrm{Mr}$. K. Kazui for calculating the differential equations. 